\title{
TAGUNG
}

\section{Die Zukunft war auch schon mal besser: Die EU im Jahr 2020}

\author{
Frédéric Krumbein*
}

Die Konferenz „Die Europäische Union 2020: Entwicklungsperspektiven der Integration“ fand im Rahmen des deutsch-französischen Forschungsprojekts „Entwicklung Deutschlands und Frankreichs bis 2020" statt. Das Ziel der Konferenz war es, mögliche Entwicklungsperspektiven der Europäischen Union zu erörtern. Dabei stand die Rolle Frankreichs und Deutschlands im Vordergrund.

Die heutigen Grundlagen künftigen europäischen Handelns

Andreas Marchetti stellte den vertraglichen Rahmen der europäischen Integration dar. Der Vertrag von Lissabon sei die neue Grundlage der Europäischen Union und nach einem langen und schwierigen Prozess ratifiziert worden. Seit 2000 gebe es bereits eine intensive Debatte über die künftige Gestalt Europas. Zu beantwortende Fragen in der Debatte seien: „Wofür brauchen wir die Europäische Union?“ (Finalität) und „Welcher vertraglicher Änderungen bedarf es, damit Europa besser funktioniert?"“ (Effizienz). Den ersten Versuch zur Beantwortung dieser Fragen stelle der Vertrag über eine Verfassung für Europa (Verfassungsvertrag) dar. Der Vertrag von Lissabon sei als Folge des gescheiterten Verfassungsvertrags entstanden.

Der Vertrag von Lissabon versuche drei Probleme zu lösen: erstens, das Demokratiedefizit in der Europäischen Union zu überwinden; zweitens, Lücken des bisherigen Primärrechts zu schließen, wie die Stimmverteilung im Rat oder die Zahl der EU-Kommissare; und drittens, die Berücksichtigung unterschiedlicher

\section{Die Europäische Union 2020: Entwicklungsperspektiven der Integration}

Tagung des Arbeitskreises Europäische Integration e.V., des Zentrums für Europäische Integrationsforschung und des Institut français des relations internationales

Mit Unterstützung der

Europäischen Kommission und der Deutschen Forschungsgemeinschaft

Bonn, 5./6. Oktober 2009

\section{Begrüßung}

Dr. Andreas MARCHETTI, Zentrum für Europäische Integrationsforschung, Bonn

Die heutigen Grundlagen künftigen europäischen Handelns

Vorsitz: Nele WISSMANN, Institut français des relations internationales, Paris

„Europa auf dem Papier“ - der vertragliche Rahmen

Dr. Andreas MARCHETTI, Zentrum für Europäische Integrationsforschung, Bonn

„Europa in den Köpfen“ - politische Vorstellungen von Meinungsbildnern und Entscheidungsträgern

Dr. Claire DEMESMAY, Deutsche Gesellschaft für Auswärtige Politik e.V., Berlin

Reaktion: Dr. Julien THOREL, Universität Saint-Etienne

Die Möglichkeiten politischer Vorhersagen - Zwischen Kaffeesatz und Empirie Vorsitz: Dr. Andreas MARCHETTI, Zentrum für Europäische Integrationsforschung, Bonn

Dr. Romain KIRT, Ministère d'Etat, Luxemburg

Rainer BREUL, Auswärtiges Amt, Berlin

\footnotetext{
* Frédéric Krumbein, Geschäftsführer des Arbeitskreises Europäische Integration e.V., Berlin.
} 
Positionen und Interessen bei politischen Entscheidungsprozessen zu befördern. Zur Überwindung des Demokratiedefizits und zur Gewährleistung der Berücksichtigung vieler Interessen werde das Europäische Parlament deutlich gestärkt. Das Parlament spiele eine stärkere Rolle im Legislativprozess. Eine weitere Neuerung sei die Möglichkeit der Europäischen Bürgerinitiative. Weiterhin habe es institutionelle Reformen gegeben. Die dreifache Mehrheit des Vertrags von Nizza ${ }^{1}$ werde abgeschafft und durch eine doppelte Mehrheit ersetzt, die aus 55 Prozent der Mitgliedstaaten und 65 Prozent der EU-Bevölkerung bestehe. Mehrheitsentscheidungen würden zum Regelfall in der europäischen Rechtsetzung. Schließlich ziele der Vertrag von Lissabon darauf, die Integration möglichst vieler unterschiedlicher Interessen und Positionen zu gewährleisten. Viele Elemente des Vertrags von Lissabon seien aus dem Verfassungsvertrag übernommen und dann in die alte Vertragsstruktur der EU-Verträge rückübersetzt worden.

Claire Demesmay sprach anschließend über die Vorstellungen von politischen Entscheidungsträgern über Europa. Sie hat für ihre Analyse Interviews mit Politikern aus Deutschland und Frankreich während der französischen EU-Ratspräsidentschaft 2008 geführt. Ihre Untersuchung kam zu drei Ergebnissen.

Erstens, die meisten Entscheidungsträger hätten nicht den Eindruck, dass die Europäische Union sich in einer Krise befinde. Viele Verantwortliche äußerten, dass die europäische Integration eine Abfolge von Krisen darstelle. Krisen hätten die Europäische Union vorangebracht. Zweitens, die Entscheidungsträger seien alle pragmatisch eingestellt gewesen. Die institutionellen Fragen seien nicht die entscheidenden Probleme für die Funktionsweise der Europäischen Union, sondern die Verhaltensweisen der einzelnen Mitgliedstaaten.
Perspektiven der Entwicklung der EU 2020 Vorsitz: Dirk FOREMNY, Zentrum für Europäische Integrationsforschung, Bonn

Die staatlich-institutionellen Konstituenten der Union

Jean QUATREMER, La Libération, Brüssel

Das politische Europa

Jared SONNICKSEN, Universität Bonn

Soziale Perspektiven

Dr. Sabine VON OPPELN, Freie Universität Berlin

Reaktion: Christiane WOLTERS, Deutsche Welle, Bonn

\section{Perspektiven der Entwicklung des europä-} ischen Umfelds 2020

Vorsitz: Dr. Louis-Marie CLOUET, Institut

français des relations internationales, Paris

Geopolitische Entwicklungspfade

Prof. Dr. Stefan FRÖHLICH, Universität

Erlangen-Nürnberg

Weltwirtschaftliche Entwicklungen

Jacques MISTRAL, Institut français des relations internationales, Paris

Umwelt und Energie

Cécile KÉRÉBEL, Institut français des relations internationales, Brüssel

Reaktion: Dr. Thomas DEMMELHUBER, Universität Erlangen-Nürnberg

\section{Schlussbemerkungen}

Dr. Louis-Marie CLOUET, Institut français

des relations internationales, Paris

Dr. Andreas MARCHETTI, Zentrum für

Europäische Integrationsforschung, Bonn

Drittens, es gebe keinen europäischen Traum, keine große europäische Idee mehr. Es gebe aber weiterhin wichtige europäische Werte, wie Menschenrechte und den Rechtsstaat. Eine gemeinsame europäische Außen- und Sicherheitspolitik sei ein von beiden Seiten oft genanntes gemeinsames Projekt. Frankreich und Deutschland hätten in diesem Bereich

1 Der Begriff der dreifachen Mehrheit meint eine gleichzeitige Mehrheit der Mitglieder des Rates, der gewichteten Stimmen der Mitglieder des Rates sowie die Erfüllung der Bedingung, dass die Mehrheit 62 Prozent der Unionsbürger repräsentiert. 
eine ähnliche Meinung. Ein großer Unterschied zwischen Frankreich und Deutschland bestünde in der Wirtschafts- und Finanzpolitik. Die französische Bevölkerung sei für eine stärkere Koordination in der Wirtschaftspolitik. Die deutsche Bevölkerung stünde dem eher ablehnend gegenüber.

Nach Ansicht von Julien Thorel sei das französische Volk sehr schlecht über den Inhalt des Verfassungsvertrags informiert gewesen. Die französischen Wähler hätten sich während des Referendums über den Verfassungsvertrag 2005 durch die Kampagnen der Parteien und der Medien stark beeinflussen lassen. Während der Debatte über den Verfassungsvertrag sei das beherrschende Bild der „polnische Klempner“ (,plombier polonais“) gewesen, der französische Arbeitsplätze bedrohe. Das Bild eines liberalisierten und für den einzelnen Bürger bedrohlichen Europas hätte die Debatte dominiert. Franzosen fühlten sich weiterhin in erster Linie als Franzosen und nicht als Europäer. Sie wollten in der Europäischen Union ein großes Frankreich wiederfinden, stärker als Deutschland ein großes Deutschland wünsche. Die nationalen Probleme würden immer auf Europa abgeschoben. Es werde aber nie über die Erfolge Europas gesprochen. So seien die Rolle des französischen Präsidenten Nicolas Sarkozy während der Georgienkrise und während der Finanzkrise nicht als Erfolge Europas gesehen worden, sondern als Erfolge Frankreichs.

In der anschließenden Diskussion bemerkte Andreas Marchetti, dass der Vertrag von Lissabon mindestens für die nächsten zehn Jahre bestehen bleiben werde. Die entscheidende Frage sei, wie der Vertrag mit politischem Leben gefüllt werde. Der Vertrag bilde nur den Rahmen des politischen Handelns in Europa.

Die Möglichkeiten politischer Vorhersagen zwischen Kaffeesatz und Empirie

Romain Kirt bemerkte zu Beginn seines Vortrages, Politiker seien immer an politischen Vorhersagen interessiert. Die künftige Ent- wicklung lasse sich aber nicht voraussagen. Trotzdem gebe es sich erfüllende Vorhersagen.

In Europa gebe es eine Aversion gegenüber Zukunftsszenarien und große Zukunftsängste. Die europäische Gesellschaft sei im Gegensatz zur amerikanischen Gesellschaft eine Angstgesellschaft. Amerika sei eine Risikogesellschaft. Europa lebe gut mit seinem Sozialstaat und habe Angst vor einem Abbau seines Wohlstands als Folge der Globalisierung. Diese Ängste hätten eine große Rolle bei der Ablehnung des Verfassungsvertrags gespielt. Dabei sei die Globalisierung eine Entwicklung, die ihren Ursprung in Europa habe (zum Beispiel die spanischen und englischen Weltreiche früherer Zeiten). Die Globalisierung sei vergleichbar mit dem Gesetz der Schwerkraft, man könne nicht dafür oder dagegen sein.

Europa schaue zudem mehr in die Vergangenheit als in die Zukunft. Ein Blick in die Vergangenheit schade nie, wenn man die Zukunft voraussagen wolle. Wer nicht intensiv über seine Vergangenheit nachdenke, werde keine Zukunft haben.

Bei Prognosen agiere der Wissenschaftler als Ratender und nicht als Wissender. Trotzdem bleibe die Zukunft nicht zwangsläufig ein weißer Fleck, da sie von uns in der Realität schon geformt und vorgestaltet werde. Wie müssten Zukunftsszenarien beschaffen sein, um Gehör zu finden? Sie sollten plausibel und realisierbar sein. Weiterhin sollten Prognosen keine abgewandelten Versionen bereits existierender Szenarien darstellen, sondern bestehende Paradigmen hinterfragen. Um wahrgenommen zu werden, müsse ein Szenario zudem von allgemein anerkannten Normen und Regeln abweichen.

Rainer Breul sprach über die Schwierigkeit Handlungsempfehlungen für künftiges politisches Handeln zu geben. Die Zukunft unterteilte Breul anhand von zwei Kategorien: „known unknowns“ und ,unknown unknowns“. Bei den 
„known unknowns“ seien bestimmende Faktoren der Zukunft bekannt, wie Energieknappheit oder der Klimawandel. Wir wüssten, dass es diese Faktoren gebe und weiter geben wird, aber wir kennen ihre Auswirkungen nicht.

Die „unknown unknowns“ stellten Zukunftsthemen dar, die vollkommen unbekannt seien und plötzlich auftauchten. Ein Beispiel sei der Tsunami in Asien im Jahr 2004. Bis zum Jahr 2004 hätten nur wenige Experten gewusst, dass es solche Riesenwellen überhaupt gebe. Ein Beispiel für die eingeschränkte Prognosefähigkeit von Extrapolationen gegenwärtiger Trends erläuterte Breul am Beispiel von Truthähnen. Wenn Truthähne sich ein Bild über die Menschen machen müssten, würde dieses positiv ausfallen: Sie werden von den Menschen gefüttert und tierärztlich versorgt. Erst am Tag der Schlachtung, der aus Sicht der Truthähne aufgrund der bisherigen Erfahrungen nicht voraussagbar sei, müssten die Truthähne alle bisherigen Annahmen schlagartig korrigieren.

Der Planungsstab des Auswärtigen Amtes in Berlin arbeite mit diesen beiden Kategorien der „known unknowns“ und der ,unknown unknowns". Er könne ebenfalls nur bestehende Trends extrapolieren und mit externen Experten über mögliche Zukunftsszenarien diskutieren.

Das wichtigste „known unknown“ in Europa sei die Implementierung des Vertrags von Lissabon. Breul sah dabei drei Prioritäten: Erstens, die Realisierung einer gemeinsamen europäischen Außenpolitik; zweitens, die Umsetzung des Prinzips der Subsidiarität; und drittens, mehr öffentliche Debatten über Europa und die Finalität der europäischen Integration.

\section{Perspektiven der Entwicklung der EU 2020}

Nach Ansicht von Jean Quatremer hat die Finanzkrise Europa maßgeblich beeinflusst und wird auch weiterhin die Zukunft Europas prägen. Dabei drohe als „,dunkles“ negatives Szenario eine Renationalisierung der Wirtschafts- politik in der Europäischen Union. Die Rettung bestimmter Industrien, wie der Automobilindustrie, sei ein Beispiel für diese Renationalisierung der Politik. Der Euro als gemeinsame Währung sei ebenfalls in Gefahr. Deutschland habe ohne jede Konsultation mit den anderen Eurostaaten beschlossen, ab 2016 auf Bundesebene und ab 2020 auf Länderebene keine neuen Schulden zu machen. Frankreich habe im Gegensatz dazu den Stabilitätspakt für sich selbst praktisch ausgesetzt.

Das ,graue“ Szenario sehe einige Abschwächungen gegenüber dem negativen „dunklen“ Szenario vor. So könnte die nationale Industriepolitik bestehen bleiben, aber auch die europäische Wettbewerbs- und Wirtschaftspolitik. Die EU-Kommission werde kein Motor der Integration mehr sein. Die Position des Präsidenten der Kommission werde sich vermutlich mit der des Präsidenten des Europäischen Rates vereinigen. Der Euro werde mehr oder weniger gut überleben.

Das „rosige“ positive Szenario bestehe in einem föderalen Europa. Der gemeinsame Markt bleibe bestehen und man werde eine gemeinsame Sozialpolitik hinzufügen. Gemeinsame soziale Mindeststandards würden etabliert, die es erlaubten, den Sozialstaat weiter zu finanzieren und zu erhalten. Weitere Verträge würden hinzukommen und verstärkte Kooperationen in Teilbereichen der Union, wie der Innen- und Justizpolitik, etablieren.

Jared Sonnicksen analysierte aktuelle und künftige Konfliktlinien (,cleavages') innerhalb des politischen Gefüges der Union. Die erste Konfliktlinie verlaufe zwischen den Skeptikern und den Befürwortern der europäischen Integration. Diese Konfliktlinie sei innerhalb des Europäischen Parlaments und während des Wahlkampfes vor den letzten Wahlen zum Europäischen Parlament zentral gewesen. Sie werde eher noch stärker werden. Weitere Konfliktlinien verliefen entlang folgender Achsen: rechtes versus linkes politi- 
sches Spektrum, kleine versus große Mitgliedstaaten, reiche versus arme Mitgliedstaaten, neue versus alte Mitgliedstaaten, neoliberale versus wohlfahrtsstaatliche Ausrichtung der Staaten sowie transatlantische Orientierung versus eigener europäischer Weg.

Die europäische Sozialpolitik war das Thema des Vortrags von Sabine von Oppeln. Die Sozialpolitik sei über Jahrzehnte ein Stiefkind europäischer Politik gewesen und habe nur über wenige Instrumente, wie im Bereich der Gleichberechtigung, verfügt. Erst seit den 1990er Jahren gebe es einen Ausbau der europäischen Sozialpolitik, insbesondere ihrer Zielsetzung. So seien die sozialen Rechte und die soziale Inklusion als Ziele auf der europäischen Ebene verankert worden. In der europäischen Sozialpolitik seien teilweise Mehrheitsentscheidungen und ein sozialer Dialog eingeführt worden. Die Sozialpolitik sei ein wichtiges Feld der europäischen Politik geworden. Der Bereich der Arbeitnehmerrechte bilde nach wie vor den Kern der gemeinsamen Sozialpolitik. Es gebe eine breite Debatte über die künftige Handlungsfähigkeit des europäischen Mehrebenensystems auf dem Gebiet der Sozialpolitik. Hier schwankten die künftigen Szenarien zwischen dem „rosigen“ Szenario einer starken europäischen Sozialpolitik mit umfassenden Kompetenzen und dem negativen Szenario einer Rückübertragung der Sozialpolitik auf die Mitgliedstaaten verbunden mit einem liberalen und sozial wenig regulierten gemeinsamen Binnenmarkt.

Der Sozialstaat stoße angesichts vielfältiger sozialer Probleme in Europa, wie einer Marginalisierung der Unterschicht oder der Kinderarmut, an seine Grenzen. Es bestehe entsprechend die Notwendigkeit eines Ausbaus der europäischen Sozialpolitik. Die soziale Kohäsion und die internationale Wettbewerbsfähigkeit Europas hingen von einem sozial und ökologisch nachhaltig erfolgreichen System $a b$.
In der Diskussion erklärte Quatremer, dass der Bürger nicht die entscheidende Kraft der europäischen Integration gewesen sei. Die Eliten hätten Europa konstruiert und dies sei nicht negativ. Die Eliten handelten im Interesse der Bürger und hätten nach dem Zweiten Weltkrieg das europäische Projekt des Friedens vorangetrieben.

\section{Perspektiven der Entwicklung des europä-} ischen Umfelds 2020

Stefan Fröhlich sprach über geopolitische Trends außerhalb der Europäischen Union. In den USA sei der unipolare Moment der Vereinigten Staaten vorbei. Die Welt werde multipolarer. Als Gründe für eine zunehmend multipolare Welt benannte er Probleme der USA, wie die Kriege im Irak und Afghanistan sowie die Wirtschafts- und Finanzkrise und den gleichzeitigen wirtschaftlichen Aufstieg Chinas. Die Vereinigten Staaten würden aber weiterhin eine Führungsrolle in einer multipolaren Welt inne haben. Der Wille zur Gestaltung und zur Führung sei noch vorhanden. Die Machtressourcen der USA, wie eine günstige demografische Entwicklung, große natürliche Ressourcen und eine hohe Anziehungskraft für Zuwanderer, würden sehr groß bleiben. Das liberale Modell der USA werde durch autoritäre Modelle wie jene Russlands und Chinas herausgefordert. Russland verfüge vor allem mit seinen Energiereserven, seinen Nuklearwaffen und einem Sitz im Sicherheitsrat der Vereinten Nationen über Machtressourcen. Russland fehle aber die ökonomische Basis, um die USA ernsthaft herauszufordern. Europa konzentriere sich auf die geopolitische Herausforderung durch Russland. Die USA würden eher China als potenzielle Bedrohung betrachten.

Die globalen wirtschaftlichen Entwicklungen analysierte Jacques Mistral. Aufgrund der weltweiten Finanz- und Wirtschaftskrise sah Mistral die liberale Ideologie der USA im Niedergang. Russland werde sich im Zeitraum von 2020 bis 2025 in einer wirtschaftlich desolaten Lage befinden. Die demografi- 
sche Entwicklung berge Probleme, die Marktwirtschaft sei schlecht implementiert und die Zukunftssektoren seien wenig entwickelt. Der einzige ökonomische Machtfaktor sei das russische Öl und Gas. Japan prognostizierte er ebenfalls einen wirtschaftlichen $\mathrm{Ab}$ stieg. China werde große Erfolge im Bereich der Bildung und der Urbanisierung machen. Trotzdem werde China bis 2020 ein Schwellenland bleiben und die Kluft zu den USA und zu Europa werde weiter bestehen. Die USA würden nach wie vor viele Vorteile im globalen Wettbewerb vorweisen können: Hervorragende Forschungszentren und Bildungseinrichtungen, einen stabilen Rechtsstaat, große natürliche Ressourcen und ein gut ausgebildetes Humankapital. Die Europäische Union werde ein wirtschaftlicher Gigant bleiben, aber ohne die natürlichen Ressourcen und ohne die funktionierenden politischen Institutionen der Vereinigten Staaten von Amerika. Die ökologische Nachhaltigkeit stelle die zentrale Herausforderung für das globale wirtschaftliche Wachstum der Zukunft dar.

Cécile Kérébel referierte über den globalen Klimaschutz. Die Europäische Union verfüge über ehrgeizige Ziele zur Reduktion ihrer $\mathrm{CO}_{2}$-Emissionen. 2005 sei der europäische Emissionshandel eingeführt worden. Seit 2008 gebe es einen EU-Plan zur Reduktion der $\mathrm{CO}_{2}$-Emissionen bis 2020 um 20 Prozent und zur Erhöhung des Anteils der erneuerbaren Energien an der europäischen Energiever- sorgung auf 20 Prozent. Der globale Anteil der Europäischen Union an den $\mathrm{CO}_{2}$-Emissionen verringere sich aber. Das größte Wachstum der $\mathrm{CO}_{2}$-Emissionen werde von China, Indien und den anderen Entwicklungsländern ausgehen. Insofern seien die Pläne der Europäischen Union nicht so entscheidend für die globale Reduktion der $\mathrm{CO}_{2}$-Emissionen. China werde 2020 voraussichtlich für 25 Prozent der $\mathrm{CO}_{2}$-Emissionen in der Welt verantwortlich sein. Es sei wahrscheinlich, dass die künftige Klimapolitik in erster Linie von den USA, von China und den anderen Entwicklungsländern bestimmt werde. Die Europäische Union könne nur über eine globale Vorbildfunktion Einfluss nehmen. Voraussetzung dafür sei aber, dass die Mitgliedstaaten den ehrgeizigen Plänen der EU-Kommission zur Reduktion der $\mathrm{CO}_{2}$-Emissionen folgen würden.

Die EU bis 2020: keine weiteren Reformen und keine Bestimmung der Finalität Europas

Abschließend bemerkten Andreas Marchetti und Louis-Marie Clouet, dass es schwierig sei, Voraussagen über Entwicklungen in der Europäischen Union bis $2020 \mathrm{zu}$ treffen. Zumindest könne man prognostizieren, dass die Zeit der institutionellen Reformen in der Europäischen Union für die nächsten zehn Jahre vorbei sei. Die Frage nach der Finalität der Europäischen Union bleibe unbeantwortet. 